\title{
Mesure de l'activité nitrate réductase durant le cycle cultural du soja (Glycine max L Merr). Répartition dans la plante et relation avec l'activité nitrogénase
}

\author{
A Kimou 1, M Obaton 2, JJ Drevon 2* \\ 1 ENSA, département d'agronomie, BP 1313, Yamoussoukro, Cóte d'lvoire ; \\ 2 INRA, laboratoire de recherches sur les symbiotes des racines, 1, place Viala, F34060 Montpellier cedex 1, France
}

(Reçu le 12 novembre 1992 ; accepté le 26 août 1993)

\begin{abstract}
Résumé - Les 2 voies de nutrition azotée, assimilation du nitrate et fixation de l'azote de l'air, ont été étudiées chez le soja (cv Hodgson), inoculé avec Bradyrhizobium japonicum USDA 138. L'évolution des 2 activités enzymatiques, nitrate réductase et nitrogénase, au cours du cycle de développement du soja en serre et au champ, indique que l'activité nitrate réductase est maximale au début du cycle et localisée dans les feuilles du sommet de la plante tandis qu'après la floraison cette activité est répartie sur l'ensemble des feuilles, sa distribution entre les étages foliaires variant suivant les conditions de culture et l'âge des plantes. De plus, durant la formation des gousses, l'activité nitrate réductase des racines et des nodosités peut contribuer significativement à la nutrition azotée puisqu'elle représente environ $25 \%$ de l'activité nitrate réductase totale de la plante. Notre travail montre également que l'assimilation de l'azote du sol et la fixation de l'azote de l'air peuvent avoir lieu simultanément. Cette complémentarité suggère qu'une amélioration substantielle du rendement pourrait être obtenue en déterminant les conditions optimales de fonctionnement de ces 2 voies de nutrition azotée chez la légumineuse nodulée.
\end{abstract}

Glycine max = soja / nitrate / nodosités / Rhizobium / symbiose

Summary - Measurement of nitrate reductase activity during the soybean (Glycine max L Merr) growth cycle: distribution in the plant and relation with nitrogenase in soybean. The 2 ways of nitrogen nutrition, nitrate assimilation and dinitrogen fixation, were studied with soybean ( $\mathrm{Cv}$ Hodgson), inoculated with Bradyrhizobium japonicum USDA 138. The evolution of the 2 enzymatic activities, nitrate reductase and nitrogenase, during the soybean growth cycle in glass-house and in the field, showed that the nitrate reductase activity was maximum during the first stages of the cycle and was located in the top leaves of the plant. After the flowering stage, this activity was distributed in all the leaves, and its distribution between the foliar levels varied according to the cultivation conditions and the plant age. Moreover, during pod formation, the nitrate reductase activity in roots and nodules can contribute significantly to nitrogen nutrition as it accounts for about $25 \%$ of total plant nitrate reductase activity. Our study also showed that soil nitrogen assimilation and atmospheric nitrogen fixation can take place simultaneously. This complementarity suggests that a substantial yield increase can be obtained through optimization of both ways of nitrogen nutrition in nodulated legumes.

Glycine max = soybean / nitrate / nodosity / Rhizobium / symbiosis 


\section{INTRODUCTION}

Au champ, les Légumineuses se développent toujours sur un milieu contenant de l'azote combiné, généralement $\mathrm{NO}_{3}{ }^{-}$. Elles peuvent donc bénéficier de deux sources d'azote, azote du sol et azote atmosphérique $\left(\mathrm{N}_{2}\right)$, lorsqu'elles sont nodulées.

Ces 2 voies de nutrition azotée, caractérisées par l'enzyme-clef de chaque voie, la nitrate réductase et la nitrogénase, ont souvent été décrites comme antagonistes (Gibson et Pagan, 1977 ; Pate et al, 1979 ; Atkins et al, 1980) parce que très coûteuses en pouvoir réducteur fourni par la photosynthèse (Salsac et al, 1984). Au champ, l'assimilation du nitrate du sol et la fixation de l'azote de l'air se succèdent généralement au cours du cycle de développement du soja (Harper, 1974 ; Thibodeau et Jaworski, 1975 ; Obaton et al, 1982), ce qui laisserait penser que ces 2 mécanismes sont exclusifs l'un de l'autre.

Afin de préciser les relations ontogéniques entre ces 2 voies de nutrition, nous avons étudié l'évolution des 2 activités enzymatiques, nitrate réductase et nitrogénase, au cours du cycle de développement du soja en serre et au champ. Par rapport à des méthodes telles que l'utilisation de ${ }^{15} \mathrm{~N}$ (Fried et Middleboe, 1977 ; Warembourg et al, 1982 ; Domenach et Corman, 1985) ou le dosage des uréides (Herridge, 1982; Zengbé et al, 1982), les mesures d'activité enzymatique permettent d'identifier des périodes de la vie de la plante où les potentiels d'activité, mais pas nécessairement les activités réelles, sont élevés, et où par conséquent, des complémentarités peuvent être utilisées pour optimiser la nutrition azotée des Légumineuses.

\section{MATÉRIEL ET MÉTHODES}

\section{Culture des plantes et des bactéries}

Le travail a été réalisé en été, avec le soja (Glycine max (L) Merr) cultivar. Hodgson (université de Minnesota, USA), inoculé avec une suspension, âgée de $7 \mathrm{j}$, de la souche de Bradyrhizobium japonicum G3 (USDA 138, Beltsville, USA) cultivée sur milieu Yem (Yeast Extract Mannitol (Vincent, 1970) à la dose de $10^{7}$ bactéries par graine.

Pour la culture en serre, les graines sont désinfectées par trempage pendant 30 min dans une solution d'hypochlorite de calcium $\left(66,7 \mathrm{~g} \mathrm{~L}^{-1}\right)$ puis rincées à l'eau stérile. Après une prégermination à l'obscurité sur vermiculite humide à $28^{\circ} \mathrm{C}$ pendant $72 \mathrm{~h}$, les plantules, de taille homogène, sont transplantées sur sable à raison de 4 plantes par pot de $5 \mathrm{~L}$ muni d'un dispositif de drainage. La solution nutritive contenant 1,5 meq $\mathrm{NO}_{3} \mathrm{LL}^{-1}$ sous forme de $\mathrm{KNO}_{3}$ est apportée par un système d'arrosage automatique à raison de $1 \mathrm{~L}$ de solution nutritive par sem et par pot. La composition de la solution nutritive est : macroéléments ( $\mathrm{mM}$ ) 3,34 $\mathrm{K}_{2} \mathrm{HPO}_{4} ; 1 \mathrm{MgSO}_{4} 7 \mathrm{H}_{2} \mathrm{O} ; 4 \mathrm{CaCl}_{2} ; 0,5 \mathrm{~K}_{2} \mathrm{SO}_{4}$ et microéléments $(\mu \mathrm{M}), 32,3 \mathrm{H}_{3} \mathrm{BO}_{3} ; 10,7 \mathrm{MnSO}_{4} \mathrm{H}_{2} \mathrm{O}$; $0,7 \mathrm{ZnSO}_{4} 7 \mathrm{H}_{2} \mathrm{O} ; 0,3 \mathrm{CuSO}_{4} 5 \mathrm{H}_{2} \mathrm{O} ; 1 \mathrm{Na}_{2} \mathrm{MoO}_{4}$ $6 \mathrm{H}_{2} \mathrm{O} ; 1 \mathrm{Co}\left(\mathrm{NO}_{3}\right)_{2} 6 \mathrm{H}_{2} \mathrm{O}$. Le fer est apporté à raison de $16,6 \mathrm{mg} \mathrm{L}^{-1}$ de versénate de fer (sequestrène Solurapide Fe 100 SG, CIBA-GEIGY SA, BP 308, 92506 Rueil-Malmaison cedex, France) contenant $50 \%$ FeEDDHA-Na dont $6 \% \mathrm{Fe}$. Le sable est rincé avec de l'eau distillée tous les $7 \mathrm{j}$ afin d'éliminer les ions en excès qui pourraient s'accumuler dans la rhizosphère. La photopériode est de $16 \mathrm{~h}$ complémentée, si nécessaire, à l'aide de lampes à vapeur de mercure de 1 $000 \mathrm{~W}\left(200 \mu \mathrm{mol}\right.$ photons $\left.\mathrm{m}^{-2} \mathrm{~s}^{-1}\right)$. La température est maintenue proche de $18^{\circ} \mathrm{C}$ pendant la nuit et de $30^{\circ} \mathrm{C}$ pendant le jour.

L'essai au champ est réalisé dans un ancien verger du domaine de l'ENSA de Montpellier. Le sol de cette parcelle est de texture argilo-sableuse, de $\mathrm{pH} 7,2$ et contient $0,10 \%$ d'azote total.

La densité au semis est de 40 graines par mètrelinéaire et les lignes sont séparées de $40 \mathrm{~cm}$. Par la suite, le peuplement est ramené à 20 plantes par mètre-linéaire $\left(510^{5} \mathrm{pl} \mathrm{ha}^{-1}\right)$. Une fumure de $10 \mathrm{~kg}$ ha $^{-1}$ d'engrais binaire $(0.18 .18)$ est apportée au moment de la préparation du sol. La parcelle est irriguée pour maintenir l'évapotranspiration maximale.

Le dispositif expérimental est en blocs de Fisher comprenant 4 répétitions et 3 traitements : non inoculé sans engrais azoté $\left(\mathrm{R}^{-} \mathrm{N}^{-}\right)$; inoculé sans engrais azoté $\left(R^{+} N^{-}\right)$; inoculé avec engrais azoté $\left(R^{+} N^{+}\right)$.

L'azote est apporté sous forme de nitrate d'ammonium $(35,5 \% \mathrm{~N})$ à la dose de $135 \mathrm{~kg} \mathrm{~N}^{-1}$, fractionnée en 9 apports de $15 \mathrm{~kg} \mathrm{~N}^{-1}$ chaque $12 \mathrm{j}$ durant les $130 \mathrm{j}$ du cycle de développement du soja. Sur le traitement non inoculé, les plantes ont présenté des symptômes visuels de carence azotée et aucune nodosité n'a été observée sur les racines, ce qui correspond à l'absence de rhizobiums spécifiques du soja dans ce sol.

\section{Mesures enzymatiques}

Les activités nitrate réductase et nitrogénase sont mesurées une fois par semaine au voisinage du midi solaire jusqu'à la maturité physiologique des gousses.

La capacité potentielle d'assimilation de l'azote nitrique est appréciée par la mesure de l'activité nitrate réductase (ARN) in vitro (Hageman et Hucklesby, 1971 ; Robin, 1979) sur un échantillon de feuille, racine ou nodosité. Au champ comme en serre, tous les prélève- 
ments sont effectués à midi. Les feuilles d'un même étage foliaire sont mélangées pour former un échantillon moyen de $1 \mathrm{~g}$ de matière fraîche (MF) représentatif de cet étage. La valeur de l'ANR foliaire par étage est obtenue en multipliant I'ANR moyenne de l'échantillon, ramenée au gramme de matière fraîche, par la masse totale de feuilles de l'étage considéré. L'ANR totale par plante est la somme des valeurs d'ANR foliaire totale des différents étages. La valeur de I'ANR totale des racines ou des nodosités est obtenue en multipliant I'ANR moyenne de l'échantillon par $g$ de matière fraîche par la masse totale des racines (sauf la partie lignifiée du pivot) ou des nodosités. La mesure de l'ANR des plantes cultivées au champ a été effectuée avec la même méthode, mais uniquement sur le système foliaire.

La fixation de l'azote moléculaire est appréciée par la mesure de l'activité réductrice d'acétylène (ARA) selon la méthode in situ. En serre, les pots, contenant les plantes, sont placés dans un sac plastique dont l'atmosphère est enrichie de 10\% d'acétylène (Balandreau et Ducerf, 1978). Au champ, un cylindre métallique est enfoncé autour de 5 pieds de soja (Dommergues et Balandreau, 1971) puis un couvercle, en matière plastique rigide laissant passer la base des tiges des plantes, est placé sur le cylindre isolant le système radiculaire dans un milieu dont l'atmosphère est enrichie de $10 \%$ d'acétylène. L'étanchéité, entre le couvercle et les tiges, est assurée par du mastic Prestik (Bostik-France SA, 77170 Coubert, France).

Bien que nous ayons vérifié au laboratoire, que le soja (cv Hodgson) ne présente pas de baisse d'activité nitrogénase en présence d'acétylène lorsqu'il est cultivé en conditions hydroponiques optimales de nutrition minérale (Drevon et al, 1988), nous ne pouvons pas assurer que la baisse d'activité nitrogénase sous acétylène, mise en évidence par Minchin et al (1986) chez le soja (cv Clark), ne soit pas présente dans les conditions de culture du soja qui ont été pratiquées dans ce travail.

La matière sèche des parties aériennes (MSA : partie de la plante située au dessus du nœud cotylédonnaire) et des nodosités (MS nod) a été déterminée par pesée après séchage à l'étuve à $75^{\circ} \mathrm{C}$ pendant $48 \mathrm{~h}$. Les analyses ont été effectuées, pour chaque traitement, sur 4 plantes pour l'ANR et 4 lots de 5 plantes pour I'ARA, la matière sèche des parties aériennes et la matière sèche des nodosités.

Les résultats sont comparés selon le test de Newman et Keuls (Dagnélie, 1973).

\section{RÉSULTATS}

\section{ANR racinaire, nodulaire et foliaire durant un cycle cultural}

La mesure in vitro, réalisée dans les conditions optimales de température, de $\mathrm{pH}$, de concentra- tion en substrats et de protection contre les inhibiteurs, permet d'apprécier la vitesse maximale de fonctionnement de l'enzyme et donc la capacité du tissu à réduire le $\mathrm{NO}_{3}$ - (Passama et al, 1989). La mesure de l'activité nitrate réductase (ANR) in vitro peut donc surestimer l'ANR réelle de la plante dans les conditions naturelles (Naik et al, 1982 ; Soussana et al, 1989). Toutefois elle permet de suivre la réduction potentielle du nitrate et d'apprécier les périodes de la vie de la plante où cette activité est importante.

Afin de déterminer la méthode d'échantillonnage pour la mesure de I'ANR totale, la première étape du travail, sur des plantes cultivées en serre, a consisté à mesurer I'ANR des feuilles sur tous les étages foliaires, ainsi que I'ANR des racines et des nodosités. Les prélèvements sont effectués à 4 stades de développement : avant la floraison (stade V4 selon Fehr et al, 1971), $30 \mathrm{j}$ après semis (JAS); au début de la floraison (V7R1), 56 JAS ; au début de la formation des gousses (V10R3), 76 JAS et pendant la formation des graines (V12R6), 96 JAS.

Les résultats obtenus (fig $1 \mathrm{~A}$ ) montrent que I'ANR totale des nodosités, exprimée par g de matière fraîche, est comparable à celle des feuilles et élevée par rapport à celle des racines, ce qui est en accord avec des observations antérieures de Hunter (1983) et de Heckmann et Drevon (1987). Mais, compte tenu de la masse des différents organes, I'ANR totale des feuilles est très supérieure à celle des parties souterraines (fig 1B), excepté lors du remplissage des graines où l'ANR des racines et des nodosités peut jouer un rôle important, puisqu'elle représente jusqu'à $25 \%$ de l'activité totale de la plante. À ce stade I'ANR décline après avoir été maximale en fin de floraison-début de formation des gousses, comme l'ont montré précédemment Harper et Hageman (1972) et Obaton et al (1982).

\section{Répartition de l'ANR entre les étages foliaires}

L'ANR des différents étages foliaires est représentée dans la figure 2. L'ANR par g de matière fraîche est maximale pour les étages foliaires les plus élevés des plantes cultivées aussi bien au champ (fig 2A) qu'en serre (fig 2B). Ces ANR élevées des feuilles du sommet peuvent être associées aux feuilles en croissance, une ANR élevée, par $g$ de matière fraîche, étant une ca- 

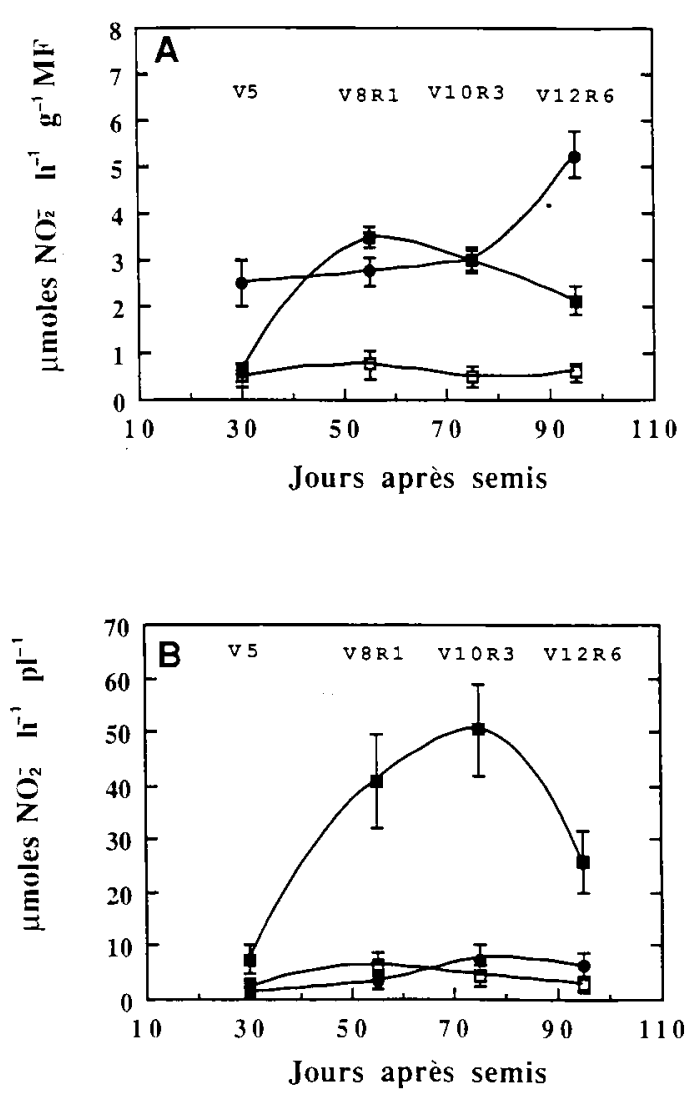

Fig 1. Évolution de l'activité nitrate réductase (ANR) au cours du cycle de développement du soja (cv Hodgson) cultivé en serre avec un apport de $1,5 \mathrm{mmol} \mathrm{NO}_{3}{ }^{-}$semaine $^{-1}$. A Activité nitrate réductase des tragments des 3 organes ( $\mu$ moles $\mathrm{NO}_{2}^{-} \mathrm{h}^{-1} \mathrm{~g}^{-1} \mathrm{MF}$ ) : $\mathbf{a}$ feuille; nodosité ; $\square$ racine. B activité nitrate réductase moyenne des 3 organes ( $\mu$ moles $\mathrm{NO}_{2}^{-} \mathrm{h}^{-1} \mathrm{pl}^{-1}$ ) : $\square$ feuille ; nodosité ; $\square$ racine. Chaque valeur est la moyenne de 4 répétitions. Les barres verticales indiquent les écart-types. MF : matière fraîche ; pl : plante. Les échantillons sont prélevés à 4 stades (V5 ; V8R1 ; V10R3 ; V12R6, selon Fehr et al, 1971).

ractéristique des jeunes organes (Tirado et al, 1990).

Il en résulte que, pour les plantes cultivées au champ, I'ANR totale par étage foliaire, ie ANR par $g$ de matière fraîche $x$ poids de matière fraîche foliaire de chaque étage, présente un maximum pour les 2 feuilles complètement déroulées sur les nœuds $n^{\circ} 10$ et 11 de la tige principale, au sommet de la plante (fig $2 A$ ). Cependant l'importance relative de l'ANR des feuilles terminales diminue avec l'âge des plantes. En effet l'ANR des 4 feuilles du sommet, qui représente plus de $80 \%$ de l'ANR totale des parties aériennes jusqu'en pleine floraison, est inférieure au quart de cette ANR totale en plein remplissage du grain (fig 3 ).
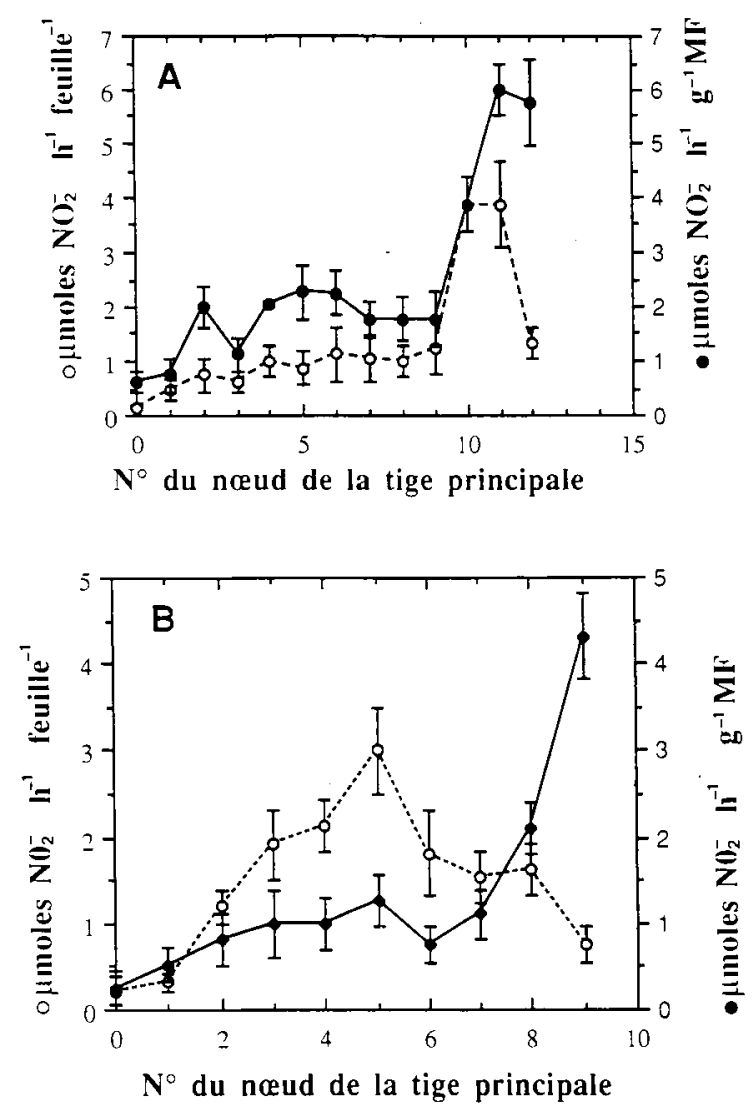

Fig 2. Répartition de l'activité nitrate réductase suivant les étages foliaires. Les plantes sont âgées de $80 \mathbf{j}$ et sont au stade de formation des gousses (R4, selon Fehr et al, 1971). () Activité nitrate réductase en $\mu$ moles $\mathrm{NO}_{2}{ }^{-h^{-1}} \mathrm{~g}^{-1} \mathrm{MF}$; activité nitrate réductase en $\mu$ moles $\mathrm{NO}_{2}{ }^{-} \mathrm{h}^{-1}$ feuille ${ }^{-1}$. A (mesure au champ) ; B (mesure en serre) chez le soja (cv Hodgson). Chaque valeur est la moyenne de 4 répétitions. Les barres verticales indiquent les écart-types. MF : matière fraîche ; pl : plante.

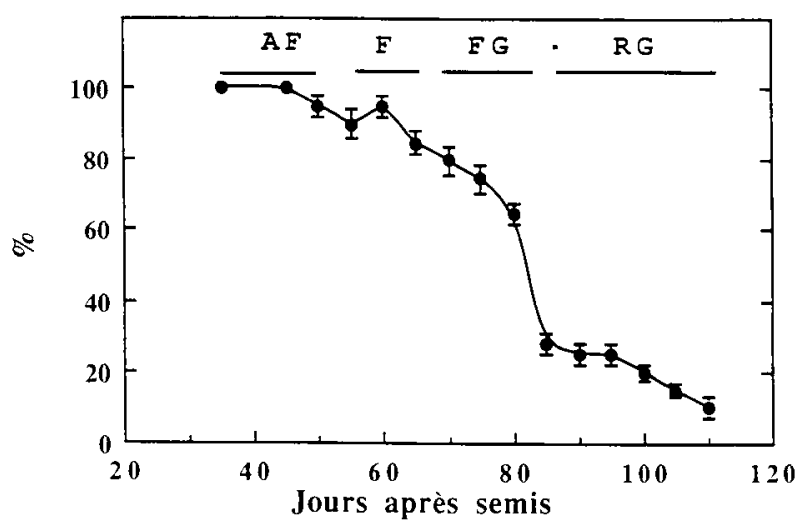

Fig 3. Évolution de l'activité nitrate réductase des 4 feuilles du sommet par rapport (\%) à l'activité nitrate réductase totale des feuilles suivant le stade de développement (Fehr et al, 1971) du soja (cv Hodgson) cultivé au champ. Chaque valeur est la moyenne de 4 répétitions. Les barres verticales indiquent les écart-types. AF : avant floraison ; $F$; floraison ; FG : formation des gousses; $R G$ : remplissage du grain. 
Pour les plantes cultivées en serre avec un ap-

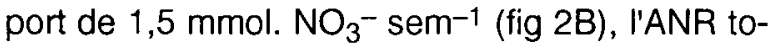
tale par étage foliaire présente un maximum aux niveaux moyens, correspondant aux nœuds $n^{\circ} 4$, 5 et 6 des tiges principales. De plus cette importance relative des feuilles médianes par rapport à celles du sommet est d'autant plus grande que les plantes sont cultivées sur une solution plus riche en nitrate (résultats non présentés).

Dans ces différentes conditions expérimentales, I'ANR des 3 folioles de chaque étage foliaire donne des valeurs très voisines (données non présentées), ce qui permet d'évaluer l'ANR d'une feuille à partir de la mesure d'activité d'une foliole par étage foliaire.

\section{Évolution de l'ANR et de l'ARA au cours du cycle végétatif}

Au champ, sans apport d'engrais azoté, l'ANR des parties aériennes (celle des parties souterraines n'étant pas prise en compte car difficilement accessible) est faible (maximum $18 \mu \mathrm{mol}$ $\mathrm{NO}_{2}-\mathrm{h}^{-1} \mathrm{pl}^{-1}$ ) et l'ARA élevée (maximum 74 $\mu$ mol $\mathrm{C}_{2} \mathrm{H}_{4} \mathrm{~h}^{-1} \mathrm{pl}^{-1}$ ) avec des pics légèrement décalés dans le temps (fig $4 A$ ).

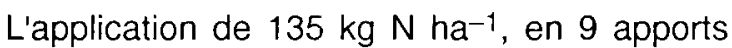
successifs, induit une activité nitrate réductase plus élevée (maximum $75 \mu \mathrm{mol} \mathrm{NO}{ }_{2}^{-} \mathrm{h}^{-1} \mathrm{pl}^{-1}$ ) et retarde l'activité nitrogénase sans en réduire l'intensité maximale $\left(74 \mu \mathrm{mol} \mathrm{C}_{2} \mathrm{H}_{4} \mathrm{~h}^{-1} \mathrm{pl}^{-1}\right)$. De plus, les périodes d'ANR et d'ARA maximales sont alors nettement séparées (fig 4B). Ce décalage est probabement dû au retard de nodulation consécutif aux premiers apports d'engrais azoté (fig 5).

Dans l'essai réalisé en serre avec un apport

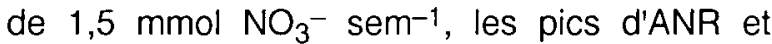
d'ARA sont superposés (fig 6 et Obaton et Kimou, 1987). Les valeurs d'ANR foliaire totale par plante (maximum de $15 \mu \mathrm{mol} \mathrm{NO}{ }_{2}^{-} \mathrm{h}^{-1} \mathrm{pl}^{-1}$ ) sont alors comparables au maximum de $20 \mu \mathrm{mol}$ $\mathrm{NO}_{2}{ }^{-\mathrm{h}^{-1}} \mathrm{pl}^{-1}$ obtenu au champ sans apport d'engrais azoté, mais plus faibles que celles du traitement azoté.

\section{Effets de l'inoculation}

Les résultats du tableau I montrent que l'inoculation multiplie par 4 le rendement en graines par rapport au témoin non inoculé et n'ayant pas
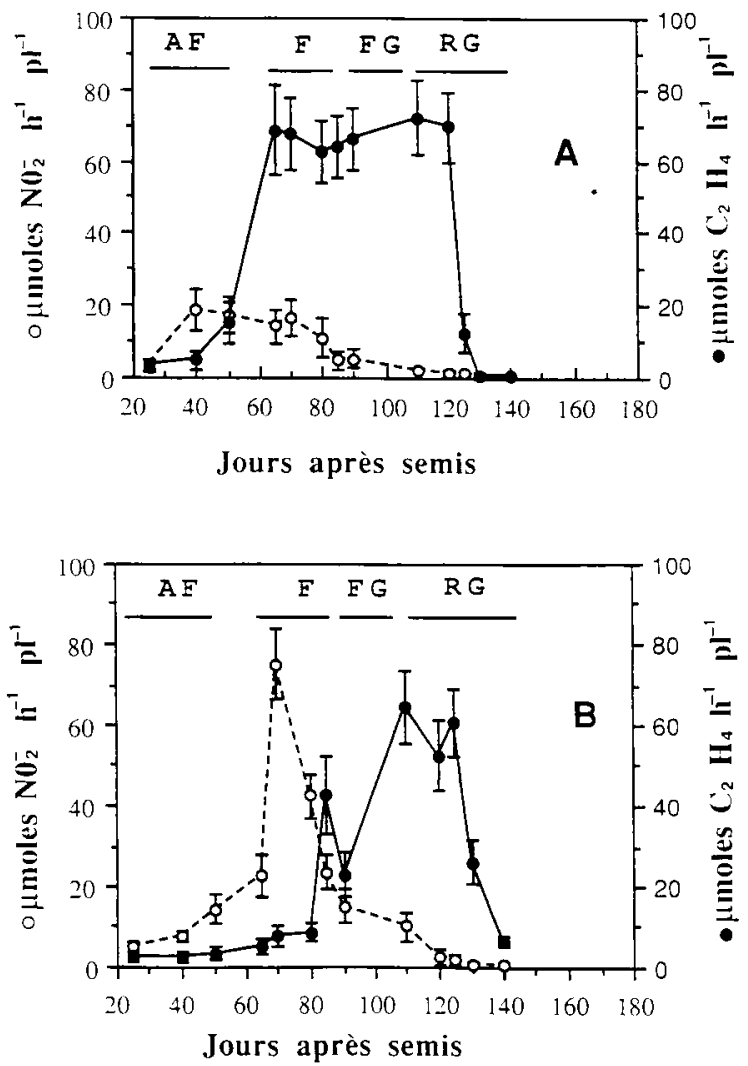

Fig 4. Évolution de l'activité nitrate réductase totale par plante $\left(0 \mu\right.$ moles $\left.\mathrm{NO}_{2}^{-} \mathrm{h}^{-1} \mathrm{pl}^{-1}\right)$ et de l'activité réductrice d'acétylène par plante $\left(\mu\right.$ moles $\left.\mathrm{C}_{2} \mathrm{H}_{4} \mathrm{~h}^{-1} \mathrm{pl}^{-1}\right)$ au champ, au cours du cycle de développement de la plante. pl : plante. (A) Parcelles témoins ne recevant pas d'engrais azoté : (B) parcelles recevant $15 \mathrm{~kg} \mathrm{~N}^{-1}$ tous les $12 \mathrm{i}$, sous forme de nitrate d'ammonium $(35,5 \% \mathrm{~N})$, au cours du cycle de développement de la plante. Chaque valeur est la moyenne de 4 répétitions. Les barres verticales indiquent les écart-types. $A F$ : avant floraison ; $F$ : floraison ; $F G$ : formation des gousses ; $R G$ : remplissage du grain.

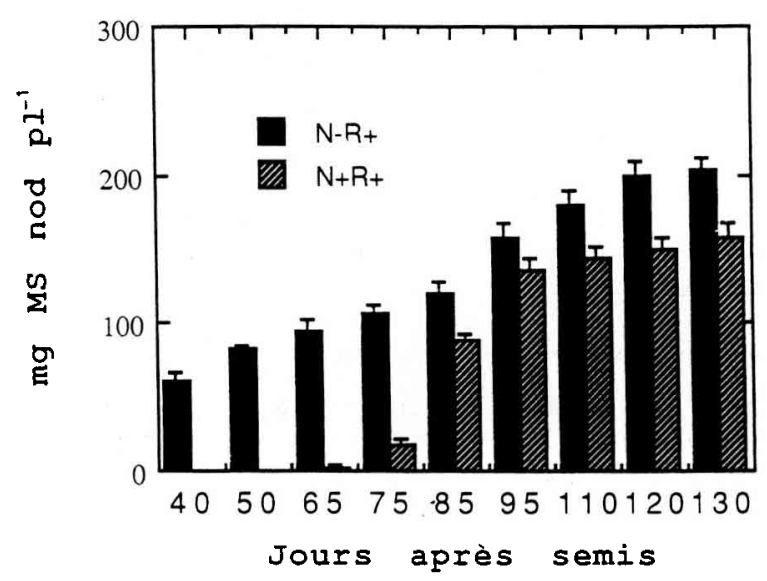

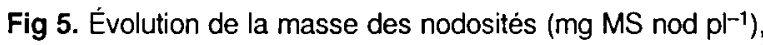
selon que les plantes inoculées $\left(R^{+}\right)$reçoivent $15 \mathrm{~kg} \mathrm{~N} \mathrm{ha}^{-1}$ tous les $12 \mathrm{j}$, sous forme de nitrate d'ammonium $(35,5 \% \mathrm{~N})$ $\left(\mathrm{N}^{+}\right)$ou pas $\left(\mathrm{N}^{-}\right)$au cours du cycle de développement du soja (cv Hodgson) cultivé au champ. Chaque valeur est la moyenne de 4 répétitions. Les barres verticales indiquent les écart-types. MS nod : matière sèche des nodosités ; $\mathrm{pl}$ : plante. 


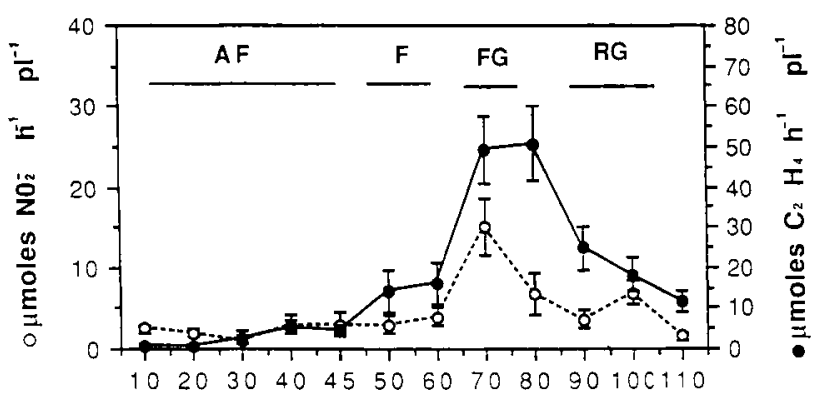

Jours après semis reçu d'engrais azoté. Ce rendement du soja inoculé n'est pas amélioré par un apport supplémentaire et fractionné de $135 \mathrm{~kg} \mathrm{~N}^{-1}$, bien que le poids des parties aériennes soit augmenté. La différence de nodulation entre les plantes témoins, inoculées et n'ayant pas reçu d'engrais azoté $\left(\mathrm{N}^{-} \mathrm{R}^{+}\right)$, et les plantes inoculées et ayant reçu l'engrais azoté $\left(\mathrm{N}^{+} \mathrm{R}^{+}\right)$(tableau $\mathrm{I}$ ) est due au retard de nodulation de ces dernières (fig 5 ), en raison, vraisemblablement, d'une concentration inhibitrice d'azote du sol au début du cycle cultural du soja dans le traitement azoté.

\section{DISCUSSION} plante (O $\mu$ mole $\mathrm{NO}_{2}^{-} \mathrm{h}^{-1} \mathrm{pl}^{-1}$ ) et de l'activité réductrice d'acétylène par plante $\left(\bullet\right.$ moles $\left.\mathrm{C}_{2} \mathrm{H}_{4} \mathrm{~h}^{-1} \mathrm{pl}^{-1}\right)$ en serre, avec un apport de $1,5 \mathrm{mmol} \mathrm{NO}_{3}^{-} \mathrm{sem}^{-1}$ au cours du cycle de développement de la plante. Chaque valeur est la moyenne de 4 répétitions. Les barres verticales indiquent les écart-types. pl : plante ; AF : avant floraison ; $F$ : floraison ; $F G$ : formation des gousses ; $R G$ : remplissage du grain.

Tableau I. Effet de l'inoculation $\left(\mathrm{R}^{+}\right)$avec la souche de Bradyrhizobium japonicum (G3) sur les paramètres de nodulation : nombre de nodosités (nod $\mathrm{pl}^{-1}$ ) ; le poids de la matière sèche des nodosités ( $\mathrm{g}$ MS nod $\mathrm{pl}^{-1}$ ) et de rendement : poids de la matière sèche des parties aériennes ( $\mathrm{kg} M \mathrm{MAS} \mathrm{ha}^{-1}$ ) et poids des graines ( $\mathrm{kg}$ graines ha- ${ }^{-1}$ ) chez le soja (cv Hogdson), cultivé au champ, en présence $\left(\mathrm{N}^{+}\right)$ou en l'absence $\left(\mathrm{N}^{-}\right)$d'engrais azoté sous forme de nitrate d'ammonium comparé à un témoin non inoculé $\left(R^{-}\right)$.

\begin{tabular}{|c|c|c|c|c|}
\hline \multirow[t]{2}{*}{ Traitements } & \multicolumn{2}{|c|}{$\begin{array}{l}\text { Nodulation } \\
\text { stade } R_{3}\end{array}$} & \multicolumn{2}{|c|}{$\begin{array}{l}\text { Biomasse Rendement } \\
\text { stade } R_{3} \text { stade } R_{8}\end{array}$} \\
\hline & $\begin{array}{c}\text { nod } \\
p t^{-1}\end{array}$ & $\begin{array}{c}g M S \text { nod } \\
p r^{-1}\end{array}$ & $\underset{h a^{-1}}{\mathrm{~kg} M S A}$ & $\underset{h a^{-1}}{k g \text { graines }}$ \\
\hline$N^{-1}$ & $O^{C}$ & $0,0 \mathrm{C}^{\mathrm{C}}$ & $4600^{c}$ & $1098^{b}$ \\
\hline $\mathrm{N}^{-} \mathrm{R}^{+}$ & $345^{a}$ & $120,75^{a}$ & $11240^{\mathrm{b}}$ & $4020^{a}$ \\
\hline $\mathrm{N}^{+\mathrm{R}^{+}}$ & $126^{b}$ & $86,94^{b}$ & $22500^{a}$ & $3660^{a}$ \\
\hline
\end{tabular}

$\mathrm{N}^{+}: 135 \mathrm{~kg} \mathrm{~N}^{-1}$ sous forme de nitrate d'ammonium $(35,5 \% \mathrm{~N})$, en 9 apports fractionnés de $15 \mathrm{~kg} \mathrm{~N}^{-1}$, tous les 12 jours depuis la levée; $\mathrm{N}: 0 \mathrm{~kg} \mathrm{~N}^{-1}$. La récolte des plantes pour l'appréciation de la nodulation et de la production de biomasse aérienne a été faite au $85^{\mathrm{e}} \mathrm{j}$ après le semis (stade $R_{3}$, Fehr et al, 1971), alors que la récolte des graines a été faite à la maturité (stade $R_{8}$ ). Dans la même colonne, les valeurs suivies de la même lettre ne diffèrent pas au seuil de $5 \%$ (Newman et Keuls).
Nous avons montré que l'ANR des parties aériennes du soja est localisée dans les feuilles du sommet de la plante en début de cycle, tandis qu'après la floraison cette activité tend à être davantage répartie sur l'ensemble des feuilles (figs 2 et 3), ce qui est en accord avec les résultats de Tirado et al (1990). De plus cette répartition dépend des conditions expérimentales puisqu'elle est différente en serre et au champ (fig 2), essentiellement parce qu'en serre les feuilles des étages médians ont une masse relativement plus élevée que celles des plantes cultivées au champ. Remarquons que les plantes cultivées en serre sont soumises à une moindre compétition pour la lumière que les plantes cultivées au champ.

En conséquence l'appréciation de I'ANR foliaire, qui représente l'essentiel de l'ANR de la plante entière, nécessite la prise en compte de tous les étages foliaires et on commettrait une erreur en extrapolant I'ANR des feuilles terminales à la plante entière. Une telle extrapolation (Harper et Hageman, 1992 ; Obaton et al, 1982 ; Conéjéro et al, 1984) conduirait en particulier, à situer trop précocement la baisse de I'ANR dans le cycle cultural.

De plus, comparée à l'ANR des feuilles qui est maximale au début du cycle, I'ANR des nodosités est relativement plus élevée durant le remplissage des grains (fig 1). Chez le soja, des vagues successives de nodulation induisent, jusqu'à un stade avancé du développement de la plante, la formation de nouvelles nodosités qui, en tant que jeunes organes, sont vraisemblable- 
ment responsables des valeurs encore élevées d'ANR nodulaire à ces stades. Nos mesures de potentiel d'ANR ne permettent cependant pas de conclure sur la contribution réelle des nodosités à la nutrition azotée du soja durant le remplissage des grains.

Notre travail montre aussi que, contrairement à certaines observations admises (Gibson et Pagan, 1977 ; Pate et al, 1979 ; Atkins et al, 1980), l'assimilation de l'azote du sol et la fixation de l'azote moléculaire peuvent avoir lieu simultanément chez le soja nodulé, comme nous l'observons en serre (fig 6). Cette superposition des activités, également observée par Harper (1976), pourrait s'expliquer par le raccourcissement du cycle végétatif de la plante à 115 j en serre, contre 135 j au champ, ainsi que par une fourniture plus faible de nitrate en serre, ce qui expliquerait également les plus faibles ANR des plantes cultivées en serre par rapport à celles de l'essai au champ.

On sait que les deux voies de nutrition peuvent être combinées efficacement en début de cycle. Harper (1974) a en effet montré qu'un apport initial d'azote en sol pauvre ou dépourvu d'azote, comme dans le cas des cultures en serre sur substrat inerte, favorise l'établissement de la symbiose et permet d'obtenir un rendement en grain plus élevé du soja que sur la base de la seule fixation d'azote moléculaire et de la réserve en azote des graines. En revanche, dans beaucoup de sols cultivés comme dans celui du témoin inoculé de notre expérience au champ, la nitrification suffirait à assurer l'approvisionnement en nitrate indispensable pour un développement initial optimal de la plante et l'établissement d'une symbiose efficace.

Nos résultats en serre suggèrent qu'il peut aussi exister une complémentarité plus tardive entre les 2 sources de nutrition azotée. Ceci n'exclut pas que ces 2 activités enzymatiques puissent être en compétition pour les substrats énergétiques (Salsac et al, 1984), du moins au niveau nodulaire (Heckmann et al, 1989), mais une telle compétition dépendrait de la dose d'apport azoté (Kimou et al, 1985). De plus, durant les stades reproducteurs, l'azote réduit serait alloué préférentiellement aux feuilles pour l'azote nitrique et aux gousses pour l'azote provenant de la fixation (Warembourg et Fernandez, 1985). Ces observations pourraient expliquer qu'une fertilisation azotée tardive améliore parfois le rendement du soja au champ (Bouniols et al, 1989).

\section{REMERCIEMENTS}

Ce travail a été soutenu par un financement CEE (DG12) dans le cadre du programme TSD 1 (Optimisation de la nutrition azotée des Légumineuses).

\section{RÉFÉRENCES}

Atkins CA, Pate JS, Griffiths J, White ST (1980) Economy of carbon and nitrogen in nodulated and non-nodulated $\left(\mathrm{NO}_{3}{ }^{-}\right.$-grown) Cowpea (Vigna unguiculata (L) Walp). Plant Physiol 66, 978-983

Balandreau I, Ducerf $P$ (1978) Activité nitrogénasique $\left(\mathrm{C}_{2} \mathrm{H}_{2}\right)$ in situ : mesure, analyse de facteurs limitants, comparaison de systèmes fixateurs d'azote. In: Isotopes in Biological Dinitrogen Fixation (International Atomic Energy Agency, ed), Vienna, 1973-188

Bouniols $A$, Blanchet $R$, Bona $S$, Cabelguenne $M$, Jones CA (1989) Modelling soybeans nitrogen nutrition in various soil depths, irrigations and fertilizations. In: IV Conf mundial de investigacion en soja (AJ Pascale, ed), Balsa de Cezeales, Buenos Aires, 2163-2170

Conéjéro G, Robin P, Salsac L (1984) Les nitrates réductases de la feuille de soja. Physiol Vég 22, 135143

Dagnelie P (1973) Théorie et méthodes statistiques. Presses Agronomiques de Gembloux, Gembloux, 107-209

Domenach AM, Corman A (1985) Use of ${ }^{15} \mathrm{~N}$ natura abundance method for the study of symbiotic fixation of field-grown soybeans. Influence of fixation on assimilation and effect of water conditions on these two functions. Soil Sci Plant Nutr 31, 311321

Dommergues $Y$, Balandreau $\mathrm{J}$ (1971) Mesure in situ de l'activité nitrogénase. GR Acad Sci Paris, série D, 273, 2020-2023

Drevon JJ, Kalia VC, Heckmann MO, Pédelahore P (1988) In situ open-flow assay of acetylene reduction activity by soybean root nodules: influence of acetylene and oxygene. Plant Physiol Biochem 26, 73-78

Fehr WR, Caviness CE, Burmood DT, Pennington JS (1971) Stage of development descriptions for soybeans, Glycine max (L) Merr. Crop Sci 11, 929-931

Fried M, Middleboe V (1977) Measurement of amount of nitrogen fixed by a legume crop. Plant Soil 47, 713-715

Gibson AH, Pagan JD (1977) Nitrate effects on the nodulation of legumes inoculated with nitrate reductase deficient mutants of Rhizobium. Planta $134,17-22$

Hageman RH, Hucklesby DP (1971) Nitrate reductase from higher plants. In: Methods in Enzymolo- 
gy (A San Pietro, ed) Academic Press, New York, vol 23, 491-503

Harper JE, Hageman RH (1972) Canopy and seasonal profiles of nitrate reductase in soybean (Glycine max L Merr). Plant Physiol 49, 146-154

Harper JE (1974) Soil and symbiotic nitrogen requirements for optimum soybean production. Crop Sci $14,255-260$

Harper JE (1976) Contribution of dinitrogen and soil or fertilizer nitrogen to soybean production. In: World Soybean Research (LD Hil, ed) The Interstate Publication, Danville, USA, 101-107

Heckmann MO, Drevon JJ (1987) Nitrate metabolism in soybean root nodule. Physiol Plant 69, 721-725

Heckmann MO, Drevon JJ, Saglio P, Salsac L (1989) Effect of oxygen and malate on nitrate inhibition in soybean nodules. Plant Physiol 90, 224-229

Herridge DF (1982) Relative abundance of ureide and nitrate in plant tissues of soybean as a quantitative assay of nitrogen fixation. Plant Physiol 70, 1-6

Hunter WJ (1983) Soybean root and nodule nitrate reductase. Physiol Plant 59, 471-475

Kimou A, Drevon JJ, Salsac L (1985) Effect de l'azote combiné sur l'efficacité relative apparente de la nitrogénase chez le soja. Physiol Vég 23, 249-256

Minchin FR, Minguez MI, Sheehy JE, Witty JF, Skot L (1986) Relationships between nitrate and oxygen supply in symbiotic nitrogen fixation by white clover. J Exp Bot 327, 1103-1113

Naik MS, Abrol YP, Nair TVR, Ramarao CS (1982) Nitrate assimilation: its regulation and relationship to reduced nitrogen in higher plants. Phytochem 21, 495-504

Obaton M, Miquel M, Robin P, Conejero G, Domenach AM, Bardin R (1982) Influence d'un déficit hydrique sur l'activité nitrate réductase et nitrogénase chez le soja. CR Acad Sci, Paris 29, 1007-1012

Obaton M, Kimou A (1987) Évolution de l'activité nitrate réductase et nitrogénase au cours du cycle végétatif chez le soja. In: Nutrition azotée des légumineuses (P Guy, ed) INRA, Versailles, 281-284

Passama L, Gojon A, Robin P, Salsac L (1987) In situ nitrate reductase activity as an indicator of nitrate availability. Plant Soil 102, 145-148

Pate JS, Layzell DB, McNeil DL (1979) Modelling the transport and utilization of carbon and nitrogen in a nodulated legume. Plant Physiol 63, 730-737

Robin P (1979) Études de quelques conditions d'extraction de la nitrate réductase des racines et des feuilles de plantules de maïs. Physiol Vég 17, 45-54

Salsac L, Drevon JJ, Zengbé M, Cleyet-Marel JC, Obaton M (1984) Energy requirements of symbiotic nitrogen fixation. Physiol Vég 22, 509-521

Sousana JF, Gojon A, Passama L, Wakrim R, Robin P (1989) Critical evaluation of the in situ nitrate reductase assay. Plant Soil 120, 243-251

Thibodeau PS, Jaworski CG (1975) Patterns of nitrogen utilization in the soybean. Planta 127, 133-147

Tirado JL, Conéjéro G, Robin P (1990) Comparaison des activités glutamine synthétase et glutamate déshydrogénase dans la feuille de Glycine max. Distribution dans la plante et effet de l'ammonium et du nitrate. Plant Physiol Biochem 28 (2), 239-280

Vincent JM (1970) A manual for the practical study of root-nodule bacteria. IBP Handbook $n^{\circ} 15$. Blackwell, Oxford, Edingbourgh, $164 \mathrm{p}$

Warembourg FR, Montange D, Bardin R (1982) The simultaneous use of $\mathrm{CO}_{2}$ and ${ }^{15} \mathrm{~N}_{2}$ labelling techniques to study the carbon and nitrogen economy of legumes grown under natural conditions. Physiol Plant 56, 46-55

Warembourg FR, Fernandez MP (1985) Distribution and remobilization of symbiotically fixed nitrogen in soybean (Glycine max) Physiol Plant 65, 281-286

Zengbé M, Obaton M, Salsac L (1982) Évolution de l'activité nitrogénase et de la teneur en uréides chez le soja (Glycine max) CR Acad Sc, Paris, 294, $1113-1116$ 